\title{
Characterizing Episodic Memory Retrieval: Electrophysiological Evidence for Diminished Familiarity following Unitization
}

\author{
Lea K. Pilgrim, Jamie G. Murray, and David I. Donaldson
}

\begin{abstract}
Episodic memory relies on both recollection and familiarity; why these processes are differentially engaged during retrieval remains unclear. Traditionally, recollection has been considered necessary for tasks requiring associative retrieval, whereas familiarity supports recognition of items. Recently, however, familiarity has been shown to contribute to associative recognition if stimuli are "unitized" at encoding (a single representation is created from multiple elements) - the "benefit" of unitization. Here, we ask if there is also a "cost" of unitization; are the ele-
\end{abstract}

\section{INTRODUCTION}

Episodic memory is supported by two independent processes-familiarity and recollection-a dissociation that is the cornerstone of dual-process theories (see Yonelinas, 2002). ERPs have proved one of the most powerful methods of dissociating the relative contributions of these processes to retrieval (Donaldson \& Curran, 2007). In particular, studies of recognition memory for words reveal two topographically and temporally distinct ERP effects that are thought to reflect familiarity and recollectionthe early bilateral frontal old-new effect and the later left parietal old-new effect, respectively (see Rugg \& Curran, 2007). In this study, we use these ERP effects to assess whether differences in the way that lexical stimuli are encoded can lead to familiarity being more or less able to support episodic memory retrieval.

Familiarity (a general sense of oldness) and recollection (recovery of contextual details) have traditionally been probed using item and associative recognition tasks, respectively. By this view, distinguishing between studied and unstudied words (item retrieval) is supported by both familiarity and recollection, whereas distinguishing between studied word pairs that are either in the same or different pairings (associative retrieval) necessarily relies on recollection. Critically, familiarity cannot support associative retrieval because, by definition, it provides no contextual information about the relationships between studied items. Recently, however, it has become clear that

University of Stirling ments of unitized representations less accessible via familiarity? We manipulated unitization during encoding and used ERPs to index familiarity and recollection at retrieval. The data revealed a selective reduction in the neural correlate of familiarity for individual words originally encoded in unitized compared with nonunitized word pairs. This finding reveals a measurable cost of unitization, suggesting that the nature of to-be-remembered stimuli is critical in determining whether familiarity contributes to episodic memory. there is no simple mapping between retrieval processes and tasks; the conditions under which familiarity and recollection are engaged is therefore open to question.

Of particular interest here is the claim that familiarity may be able to contribute to associative recognition under conditions where multiple items have been processed in a "unitized" manner (e.g., Diana, den Boom, Yonelinas, \& Ranganath, 2011; Bader, Mecklinger, Hoppstädter, \& Meyer, 2010; Rhodes \& Donaldson, 2007, 2008; Quamme, Yonelinas, \& Norman, 2007; Yonelinas, Kroll, Dobbins, \& Soltani, 1999). Unitization occurs when previously separate items are encoded as a single coherent component (cf. Graf \& Schacter, 1989). Simply stated, conditions that encourage pairs of stimuli to be unitized into a single representation allow familiarity to support retrieval because the combined representation elicits a sense of familiarity for the pairing as a whole. By this view, memory for pairs of stimuli that are processed individually (i.e., are not unitized) is more dependent on recollection; familiarity for the individual units does not provide useful information about the relationships between the parts.

The so-called "benefit" of unitization has been demonstrated across various types of stimuli, tasks, and participant groups. For example, Yonelinas et al. (1999) demonstrated greater familiarity for recognition of upright compared with inverted faces under the logic that upright faces can be treated as a unitized whole, whereas inverted faces must be processed as individual components (Searcy \& Bartlett, 1996). Unitization effects have also been demonstrated in studies using ERPs to index familiarity and recollection, for example, with word pairs related 
by preexisting associations (e.g., traffic-jam; Rhodes \& Donaldson, 2007, 2008) and unrelated word pairs joined through invented definitions (e.g., smoke-apple: a fruit maturing above flames; Bader et al., 2010). Consistent with this, the benefit of unitization has also been shown in amnesic patients who exhibit severely impaired recollection with relatively preserved familiarity. These patients show a memory advantage for unitized over nonunitized word pairs in comparison with both controls and to patients who have both recollection and familiarity impairments (Quamme et al., 2007). Taken together, these studies suggest that the unitization of multiple-part representations into a single coherent whole allows familiarity to contribute to recognition.

To date, two theoretical accounts of unitization have been proposed. The first, which is the "benefits and costs" account, argues that the process of unitizing multiple items creates a single holistic representation that is perceived and remembered as one entity (cf. Rhodes \& Donaldson, 2008; Mayes, Montaldi, \& Migo, 2007). In essence, only the unitized whole and not the constituent elements is readily available to familiarity at retrieval, and as a consequence, unitization should incur quantifiable costs when remembering the constituent elements of a unitized pair (i.e., reduced familiarity and greater reliance on recollection). An alternative possibility can be characterized as a "benefits-only" account; that is, that the process of unitization creates a unitized representation plus representations of the constituent elements. This is suggested, for example, by Yonelinas et al. (1999, p. 654) who note that, if two items are encoded as a coherent whole then, the "whole item as well as its constituent parts might increase in familiarity when studied." Although Yonelinas et al. do not elaborate further, it is clear that, by this view, the representation of each item in a word pair and the unitized representation are all accessible at retrieval, and therefore, no costs will be incurred when remembering the constituent items.

Currently, there is limited evidence that speaks to the competing accounts of unitization; however, two behavioral studies do provide relevant data. In a series of experiments, Hockley and Cristi (1996) addressed "encoding tradeoffs," that is, whether there is a cost to encoding item versus associative information when studying word pairs. In an item imagery condition, participants were encouraged to create separate images for each word (itemspecific encoding); in an interactive imagery condition, participants were asked to form interactive images or generate a sentence that included both words (associative encoding). Overall, Hockley and Cristi reported similar item and associative recognition memory irrespective of the encoding task used. By contrast, however, McGee (1980) reported quite different results across a set of four behavioral experiments using similar tasks. In this case, item recognition memory was poorer following interactive compared with item imagery, whereas associative recognition was better following interactive imagery com- pared with item imagery. Taken together, therefore, existing behavioral evidence alone does not clearly discriminate between the two theoretical accounts of unitization.

Given the lack of clear behavioral evidence, here we turn to the use of neural data to investigate the potential costs of unitization. Existing neural data are consistent with both theoretical accounts in demonstrating a "benefit" of unitization (relative to nonunitization) during associative recognition. The aim of the current experiment is to provide data where the two accounts can be dissociatedon the basis of their predictions regarding performance on item recognition. The "benefits and costs" view predicts that there should be a disadvantage for items initially encoded under unitized compared with nonunitized conditions, that is, a reduced engagement of familiarity for items from unitized compared with nonunitized pairs. In essence, the single elements of the unitized representation would not be available, whereas items encoded under nonunitized conditions would still be readily accessible. By contrast, the "benefits-only" view predicts that there would be no difference in retrieval as a function of whether items had been unitized or not during encoding-under both encoding conditions, the individual components would still be readily available and able to elicit familiarity.

The present experiment was designed to discriminate between the two competing accounts of unitization by examining potential costs during the retrieval of single words. We employed a baseline of single-item recognition, comparing it to the retrieval of items that had been initially studied within word pairs. Mental imagery was used as an encoding task throughout, but for word pair encoding, we directly manipulated unitization; following Rhodes and Donaldson (2008), encoding either encouraged (interactive imagery) or discouraged (item imagery) unitization. To measure the contribution of recollection and familiarity to retrieval, we recorded ERPs during recognition memory; participants were simply presented with single words and required to judge whether they were old (studied) or new (unstudied). The critical question was whether, in the context of identical retrieval demands, the ERP data would reveal differences in the contribution of familiarity to recognition memory as a function of unitization.

\section{METHODS}

\section{Participants}

Thirty-one University of Stirling undergraduates were paid $\$ 5 /$ hr to take part. Nine participants' data were rejected because of equipment failure, excessive EEG artifacts, or insufficient number of correct trials in at least one experimental condition. Twenty-two participants remained (12 women, average age $=20.5$ years, range $=17-$ 26 years). All were right-handed native English speakers and had normal or corrected-to-normal vision and no known language or neuropsychological impairments. Informed 
consent was collected according to University of Stirling ethics procedures.

\section{Stimuli}

Four hundred nouns and verbs were selected from the Medical Research Council database (Coltheart, 1981). Two hundred of these words were randomly divided into three study conditions: single items (40 words), nonunitized (40 word pairs), and unitized (40 word pairs). The remaining 200 were selected for presentation at test as "new" words.

Words were matched across conditions on age of acquisition, familiarity, frequency, concreteness, and imageability (see Table 1 for means and standard deviations). Visual inspection initially established that word pairs in the nonunitized and unitized conditions were unrelated. Additional analyses confirmed that pairs were not semantically related using Latent Semantic Analyses (lsa.colorado.edu) with a mean semantic relatedness rating of $0.04(S D=0.03)$. In addition, backward and forward association ratings were all less than 0.02 according to the Edinburgh Association Thesaurus (Kiss, Armstrong, Milroy, \& Piper, 1973).

\section{Procedure}

The experiment was conducted on a standard desktop PC using E-Prime software (version 1.2), and responses were recorded on a PST Serial Response box (Psychology Software Tools, Pittsburgh, PA; www.pstnet.com). Words were presented in white against a black background using lowercase, 18-point Courier New font. Single words were displayed in central vision; word pairs were presented one word above the other, slightly above and below center.

The item study condition was always presented first, followed by either nonunitized or unitized conditions (order counterbalanced across participants). Each of the three conditions was preceded by a number of practice trials, following which the experimenter was able to confirm that the participants were using the appropriate encoding strategy. Each study trial began with a fixation cross $(+)$ displayed in the center of the screen for 1000 msec. The cross was used to maintain the partici-

Table 1. Item Means (and Standard Deviations)

\begin{tabular}{lccc}
\hline & Item & Nonunitized & Unitized \\
\hline Age of acquisition & $375(97)$ & $347(95)$ & $369(101)$ \\
Concreteness & $483(110)$ & $474(114)$ & $473(127)$ \\
Familiarity & $494(69)$ & $522(66)$ & $511(64)$ \\
Imageability & $503(85)$ & $496(93)$ & $496(92)$ \\
Kučera-Francis frequency & $45(87)$ & $59(69)$ & $54(62)$ \\
Letter length & $5.60(1.59)$ & $5.29(1.30)$ & $5.57(1.43)$ \\
\hline
\end{tabular}

pants' fixation on the center of the screen and to indicate the presentation of the next word/word pair. This was followed by a 500-msec blank screen, and then, either a word or a word pair was displayed for $2000 \mathrm{msec}$. Participants were instructed to generate a single mental image (item), two separate mental images (nonunitized), or a single interacting mental image (unitized). These instructions were intended to encourage or discourage unitization of word pairs. The trial ended with a blank screen for $200 \mathrm{msec}$.

Each study condition (item, nonunitized, or unitized) was immediately followed by the test phase. During test, only single previously studied or new words were presented (for all three conditions). Two test lists were created for nonunitized and unitized conditions so that only one item of a pair was presented (to prevent cueing effects). All test trials began with a fixation cross presented for 1000 msec followed by a blank screen for 400 msec. Single words were then presented for $500 \mathrm{msec}$. The trial ended with a blank screen for $1500 \mathrm{msec}$. Participants were required to make an old-new response as quickly and accurately as possible on presentation of the target. Responses were made on a serial response box using the index/middle finger on each hand. The mapping of response buttons was counterbalanced between participants.

\section{ERP Recording}

Scalp EEG was recorded from $62 \mathrm{Ag}-\mathrm{AgCl}$ electrodes embedded in an elastic cap (Quick-Cap, Neuromedical Supplies, Sterling, VA: www.neuro.com) in accordance with an extended version of Jasper's (1958) International 10-20 system (FP1, FPz, FP2, AF3, AF4, F7, F5, F3, F1, Fz, F2, F4, F6, F8, FT7, FC5, FC3, FC1, FCz, FC2, FC4, FC6, FT8, T7, C5, C3, C1, Cz, C2, C4, C6, T8, TP7, CP5, CP3, CP1, CPz, CP2, CP4, CP6, TP8, P7, P5, P3, P1, Pz, P2, P4, P6, P8, PO7, PO5, PO3, POz, PO4, PO6, PO8, CB1, O1, Oz, $\mathrm{O} 2$, CB2; see Figure 1). Two additional electrodes, positioned on the left and right mastoids (M1, M2), served as references. Electrodes were placed above and below the left eye (vertical EOG) and on the outer canthus of each eye (horizontal EOG) to monitor eye movements and blinks. All electrodes were recorded referenced to an additional reference located midway between electrodes $\mathrm{Cz}$ and $\mathrm{CPz}$, and then rereferenced off-line to recreate an averaged mastoid reference. Electrode impedances were kept below $5 \mathrm{k} \Omega$. Recordings were made using Synamps 2 amplifier and Neuroscan 4.4 Acquire software (www.neuro. com). Signals were amplified with a gain of 2010 using a band-pass filter of $0.1-40 \mathrm{~Hz}$ and digitized at a rate of $250 \mathrm{~Hz}$.

\section{Data Processing}

The EEG data were analyzed off-line using Neuroscan 4.3 Edit software (www.neuro.com). The raw EEG was visually inspected, and segments of data were rejected if they included high levels of noise. Eye blinks were removed 
Figure 1. Schematic maps of 62 electrodes with those used for the analyses highlighted. inf $=$ inferior; mid = middle; $\sup =$ superior.

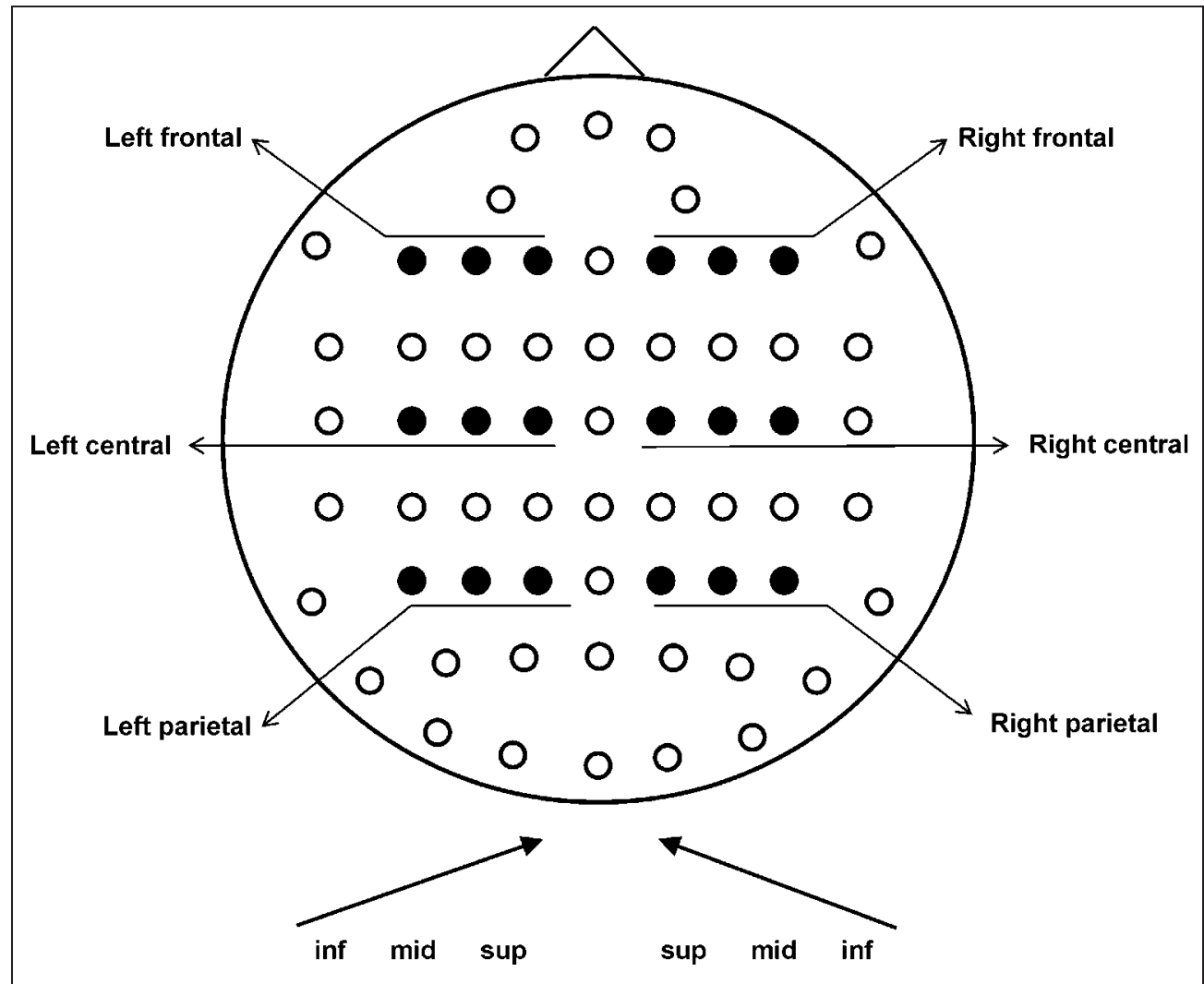

using the Neuroscan Ocular Artifact Reduction procedure (using a minimum of 32 blinks per participant). EEG data were epoched and time-locked to stimulus presentation at test using $2040 \mathrm{msec}$ time windows ( $-104 \mathrm{msec}$ to $+1936 \mathrm{msec}$ ). Epochs were rejected if they had a baseline drift exceeding $\pm 75 \mu \mathrm{V}$ or where signal change exceeded $\pm 100 \mu \mathrm{V}$. Data were smoothed over a 5-point kernel and baseline corrected with respect to the prestimulus presentation period ( $-104 \mathrm{msec}$ to $0 \mathrm{msec})$.

Epochs were sorted to create separate ERPs for "hits" (correct "old" responses to studied words) and "correct rejections" (correct "new" responses to unstudied words). Individual participant waveforms were averaged together to produce grand-averaged waveforms, with a requirement of at least 16 trials per condition from each participant. The mean number of trials contributing to each average ERP was, in the item condition, old (64) and new (67), nonunitized old (53) and new (58), and unitized old (50) and new (58). ERPs were analyzed by examining mean amplitudes during critical time windows, relative to the prestimulus baseline. Where appropriate, the Greenhouse-Geisser correction was applied, and the corrected levels of probability are reported.

\section{RESULTS}

\section{Behavioral Data}

A summary of behavioral results is provided in Table 2, indicating that recognition of words encoded in the item condition was more accurate and faster than in both the nonunitized and unitized conditions. In contrast, however, there were no differences in accuracy or speed of responses to nonunitized compared with unitized conditions. The data were analyzed using separate repeatedmeasures ANOVAs, performed on discrimination $\left(\mathrm{d}^{\prime}\right)$ and bias (see Snodgrass \& Corwin, 1988) as well as RTs for hit and correct rejection responses.

\section{Discrimination and Bias}

Analysis of $\mathrm{d}^{\prime}$ data employed an ANOVA, comparing all three Study Conditions, and confirmed that discrimination was significantly easier in the item compared with both

Table 2. d', Bias, Percent (\%) for Correct Hits/False Alarms, and Mean RTs for Hits/Correct Rejections

\begin{tabular}{lccc}
\hline & Item & Nonunitized & Unitized \\
\hline $\mathrm{d}^{\prime}$ & $.90(.10)$ & $.78(.14)$ & $.76(.15)$ \\
Bias & $.38(.27)$ & $.34(.18)$ & $.31(.18)$ \\
$\%$ (correct hits) & $.90(.10)$ & $.77(.14)$ & $.75(.15)$ \\
$\%$ (FAs) & $.07(.07)$ & $.11(.09)$ & $.11(.09)$ \\
RTs (hits) & $653(105)$ & $665(121)$ & $675(107)$ \\
RTs (CRs) & $721(109)$ & $715(111)$ & $710(91)$ \\
\hline
\end{tabular}

$\mathrm{CRs}=$ correct rejections; FAs $=$ false alarms 
nonunitized $\left(F_{1,21}=39.89, p<.0001\right)$ and unitized $\left(F_{1,21}=\right.$ $32.86, p<.0001)$ conditions. By contrast, $\mathrm{d}^{\prime}$ did not differ significantly across nonunitized and unitized conditions $\left(F_{1,21}=1.30, p=.267\right)$; as Table 2 shows, discrimination was well matched across the nonunitized and unitized conditions. Importantly, analysis of bias data revealed that there were no significant differences in response bias between the conditions ( $p>.30$ in all cases); participants were relatively conservative for all three conditions (see Table 2).

RTS

Analysis of RT data employed an ANOVA with the factors of Response (hit/correct rejection) and Study Condition (item/nonunitized/unitized) and revealed a main effect of Response $\left(F_{1,21}=62.78, p<.0001\right)$ but no main effect of Study Condition or interaction between the two. As is clear from Table 2, the effect of Response reflects the fact that hits were made significantly faster than correct rejections in all three study conditions.

\section{ERP Analyses}

The principal aim of the electrophysiological analyses was to examine the contribution of the ERP correlates of familiarity and recollection to item recognition performance following different encoding conditions. Two standard time windows were analyzed, namely, 300-500 msec capturing the early bilateral frontal old-new effect (familiarity) and 500-700 msec capturing the later left parietal old-new effect (recollection). To ensure that ERP effects of interest were adequately captured in the analyses, initial repeated-measures ANOVAs were conducted separately for each study condition, with factors of Retrieval (old/ new), Location (frontal/central/parietal), Hemisphere (left/right), and Site (inferior/middle/superior), as illustrated in Figure 1. The critical comparison was between hits and correct rejections in each encoding condition, allowing the presence of reliable old-new effects to be established in each case. The initial analyses were then followed up by additional between-task comparisons, closely targeting the familiarity and recollection effects. These analyses were performed with focused $t$ tests using an a priori selection of electrodes from bilateral frontal (F5, F3, F1, FZ, F2, F4, and F6) and left parietal (P1, P3, and $\mathrm{P} 5)$ regions of the scalp.

\section{Familiarity: Early Frontal Old-New Effects}

As can be seen from Figure 2, examination of the early time window reveals the presence of an early bilateral frontal modulation for both the item (top row) and nonunitized conditions (middle row). As the topographic maps show, the old-new differences exhibit a frontocentral distribution during the early 300-500 msec time
Figure 2. Bilateral frontal old-new effect at electrode Fz from -100 to $+1000 \mathrm{msec}$ (amplitude scale: $+5 \mu \mathrm{V}$ to $-5 \mu \mathrm{V})$. Gray box highlights 300-500 msec. Blue = old; red $=$ new. Topographic maps show the distribution of the old-new effect during 300-500 msec epoch.

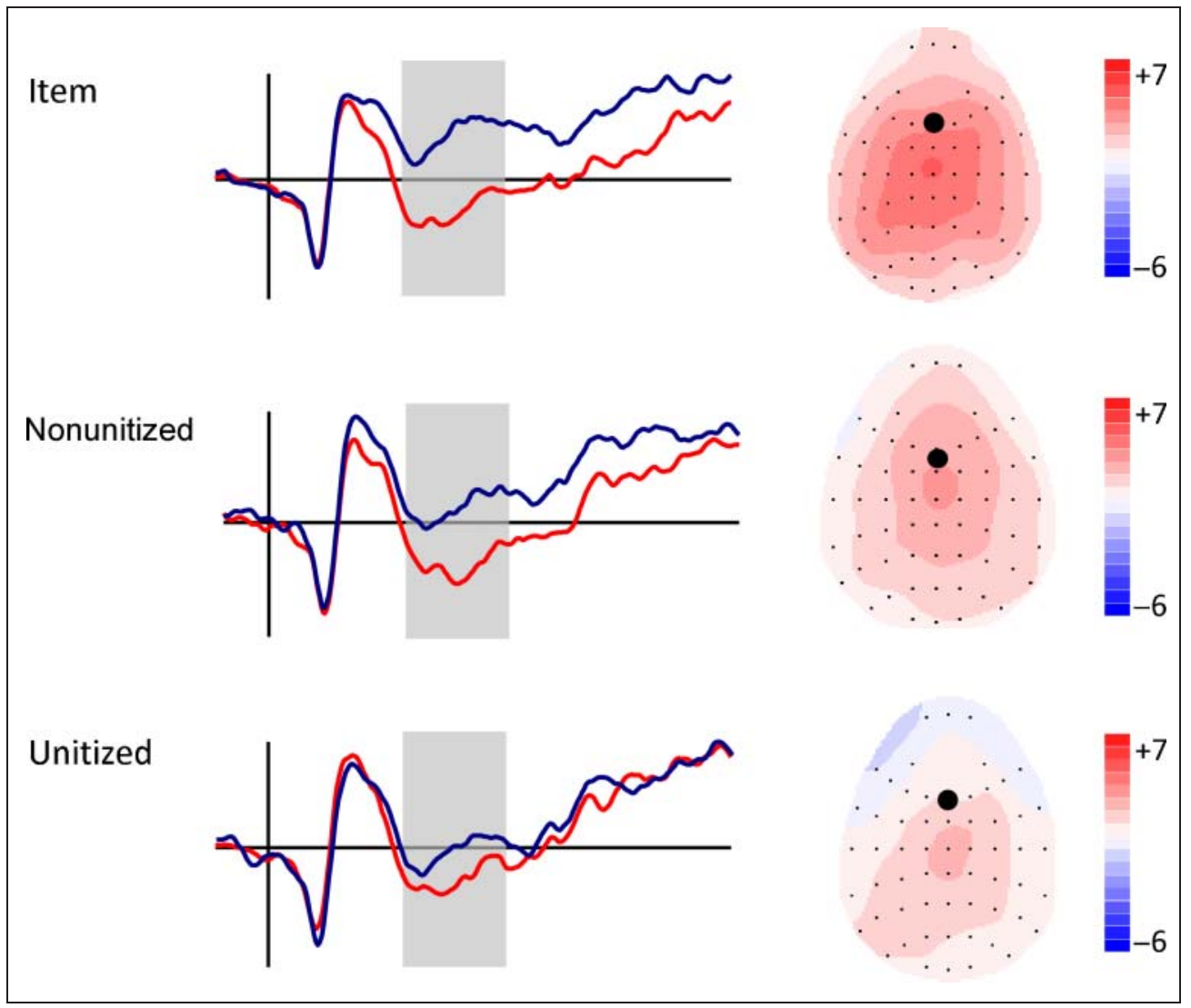


window. By contrast, however, the bilateral frontal modulation is clearly reduced in the unitized condition (bottom row), with the topographic map indicating a more centralparietal focus. When data from six scalp regions (18 electrodes highlighted in Figure 1) were entered into analysis, all three conditions showed significant main effects of Retrieval (see Table 3 for a full report of significant/marginal main effects and interactions), reflecting the fact that ERPs to hits were more positive than to ERPs for correct rejection in each case. Planned comparisons targeting frontal electrodes confirmed that the early bilateral frontal effect was highly significant in both the item $\left(t_{21}=7.10, p<\right.$ $.0001)$ and nonunitized $\left(t_{21}=6.27, p<.0001\right)$ conditions.
In the unitized encoding condition, the bilateral frontal effect was much smaller and only marginally significant $\left(t_{21}=2.04, p=.054\right)$.

Planned comparisons were carried out to directly analyze the data across study conditions, using difference waveforms (old-new) to calculate the size of the oldnew effect in each condition. To examine the size of the bilateral frontal effect, this analysis was carried out on data from frontal sites during the 300-500 msec time window (cf. Curran, 2004). The analysis confirms that the amplitude of the old-new effect was similar in item and nonunitized conditions $\left(t_{21}=1.88, p=.074\right)$ but larger for the item compared with the unitized condition $\left(t_{21}=5.00\right.$,

Table 3. Old versus New Amplitudes across All (18) Electrodes in Early and Late Time Windows

\begin{tabular}{|c|c|c|c|c|c|c|}
\hline Time Window & Latency Period & Comparison & Effect & $d f$ & $F$ & $p$ \\
\hline \multirow[t]{13}{*}{1} & 300-500 msec & Item old-new & $\mathrm{R}$ & 1,21 & 103.56 & .0001 \\
\hline & & & $\mathrm{R} \times \mathrm{S}$ & 1,22 & 39.75 & .0001 \\
\hline & & & $\mathrm{R} \times \mathrm{H} \times \mathrm{L}$ & 2,38 & 7.14 & .003 \\
\hline & & & $\mathrm{R} \times \mathrm{L} \times \mathrm{S}$ & 2,43 & 6.97 & .002 \\
\hline & & & $\mathrm{R} \times \mathrm{H} \times \mathrm{L} \times \mathrm{S}$ & 2,43 & 7.27 & .002 \\
\hline & & Nonunitized old-new & $\mathrm{R}$ & 1,21 & 48.34 & .0001 \\
\hline & & & $\mathrm{R} \times \mathrm{S}$ & 1,26 & 89.61 & .0001 \\
\hline & & & $\mathrm{R} \times \mathrm{L} \times \mathrm{S}$ & 2,44 & 7.66 & .001 \\
\hline & & Unitized old-new & $\mathrm{R}$ & 1,21 & 15.85 & .001 \\
\hline & & & $\mathrm{R} \times \mathrm{S}$ & 1,24 & 26.81 & .0001 \\
\hline & & & $\mathrm{R} \times \mathrm{H} \times \mathrm{L}$ & 2,34 & 4.88 & .019 \\
\hline & & & $\mathrm{R} \times \mathrm{L} \times \mathrm{S}$ & 2,36 & 4.53 & .022 \\
\hline & & & $\mathrm{R} \times \mathrm{H} \times \mathrm{L} \times \mathrm{S}$ & 2,44 & 4.21 & .020 \\
\hline \multirow[t]{14}{*}{2} & 500-700 msec & Item old-new & $\mathrm{R}$ & 1,21 & 44.93 & .000 \\
\hline & & & $\mathrm{R} \times \mathrm{S}$ & 1,24 & 15.75 & .000 \\
\hline & & & $\mathrm{R} \times \mathrm{L} \times \mathrm{S}$ & 2,52 & 10.52 & .000 \\
\hline & & & $\mathrm{R} \times \mathrm{H} \times \mathrm{L} \times \mathrm{S}$ & 2,33 & 2.79 & .086 \\
\hline & & Nonunitized old-new & $\mathrm{R}$ & 1,21 & 18.55 & .000 \\
\hline & & & $\mathrm{R} \times \mathrm{L}$ & 1,23 & 3.59 & .067 \\
\hline & & & $\mathrm{R} \times \mathrm{S}$ & 1,25 & 28.85 & .000 \\
\hline & & & $\mathrm{R} \times \mathrm{L} \times \mathrm{S}$ & 2,38 & 4,95 & .014 \\
\hline & & Unitized old-new & $\mathrm{R}$ & 1,21 & 6.26 & .021 \\
\hline & & & $\mathrm{R} \times \mathrm{L}$ & 1,25 & 8.89 & .005 \\
\hline & & & $\mathrm{R} \times \mathrm{H} \times \mathrm{L}$ & 1,29 & 3.43 & .062 \\
\hline & & & $\mathrm{R} \times \mathrm{S}$ & 1,23 & 12.74 & .001 \\
\hline & & & $\mathrm{R} \times \mathrm{L} \times \mathrm{S}$ & 2,44 & 4.70 & .013 \\
\hline & & & $\mathrm{R} \times \mathrm{H} \times \mathrm{L} \times \mathrm{S}$ & 1,32 & 3.34 & .060 \\
\hline
\end{tabular}

$\mathrm{R}=$ retrieval; $\mathrm{H}=$ hemisphere; $\mathrm{L}=$ location; $\mathrm{S}=$ site.

Greenhouse-Geisser corrected statistics reported where appropriate. 


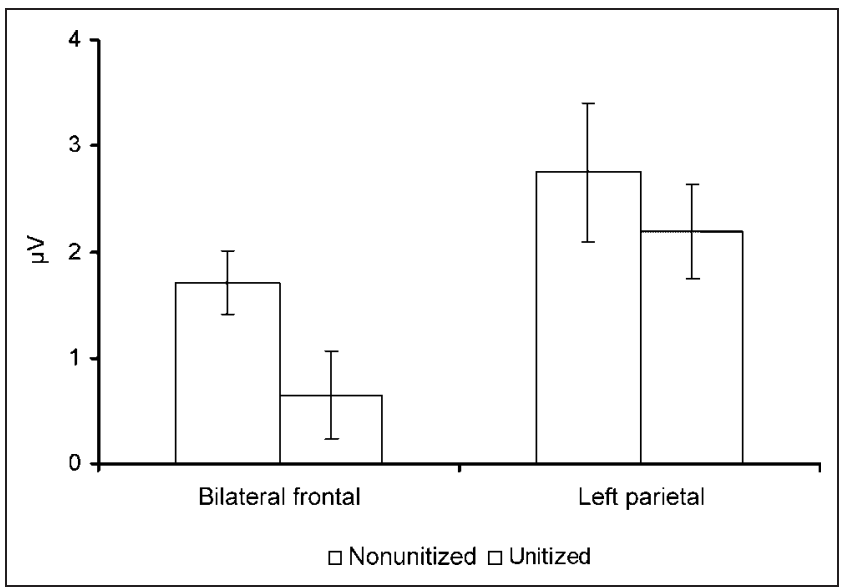

Figure 3. Bilateral frontal and left parietal old-new effects (with standard error bars).

$p<.0001)$. Critically, the effect was also significantly larger for the nonunitized than the unitized condition $\left(t_{21}=2.34, p=.031\right.$; see Figures 2 and 3$)$.

\section{Recollection: Later Left Parietal Old-New Effects}

Figure 4 illustrates the later time window; all three conditions show clear and widespread old-new differences that extend across left parietal electrodes, indicating the presence of recollection. When all 18 electrodes were entered into the analysis, all three conditions showed significant main effects of Retrieval along with significant interactions involving Retrieval, Location, and Site (see Table 3 for a full report of all main effects and interactions), indicating the posterior focus of activity in each case. Importantly, planned comparisons confirmed the presence of highly significant left parietal old-new effects in all three conditions: item $\left(t_{21}=6.74, p<.0001\right)$, nonunitized $\left(t_{21}=\right.$ $4.19, p<.0001)$, and unitized $\left(t_{21}=4.86, p<.0001\right)$.

Additional planned comparisons were used to examine the size of effects across conditions, revealing that the left parietal old-new effect was significantly larger for the item compared with both the unitized $\left(t_{21}=4.48, p<.0001\right)$ and nonunitized conditions $\left(t_{21}=4.63, p<.0001\right)$. Importantly, there was no significant difference between nonunitized and unitized conditions $\left(t_{21}=.302, p=.766\right)$ (see Figure 3 ).

\section{DISCUSSION}

The aim of the current study was to discriminate between two accounts of unitization-the "benefits and costs" and "benefits-only" views. We explicitly manipulated the way in which word pairs were encoded, allowing us to examine the ERP correlates of retrieval as a function of whether
Figure 4. Left parietal old-new effect at electrode P1 from -100 to +1000 msec (amplitude scale: $+12 \mu \mathrm{V}$ to $-4 \mu \mathrm{V})$. Gray box highlights 500-700 msec. Blue = old; red $=$ new. Topographic maps show the distribution of the old-new effect during 500-700 msec epoch.

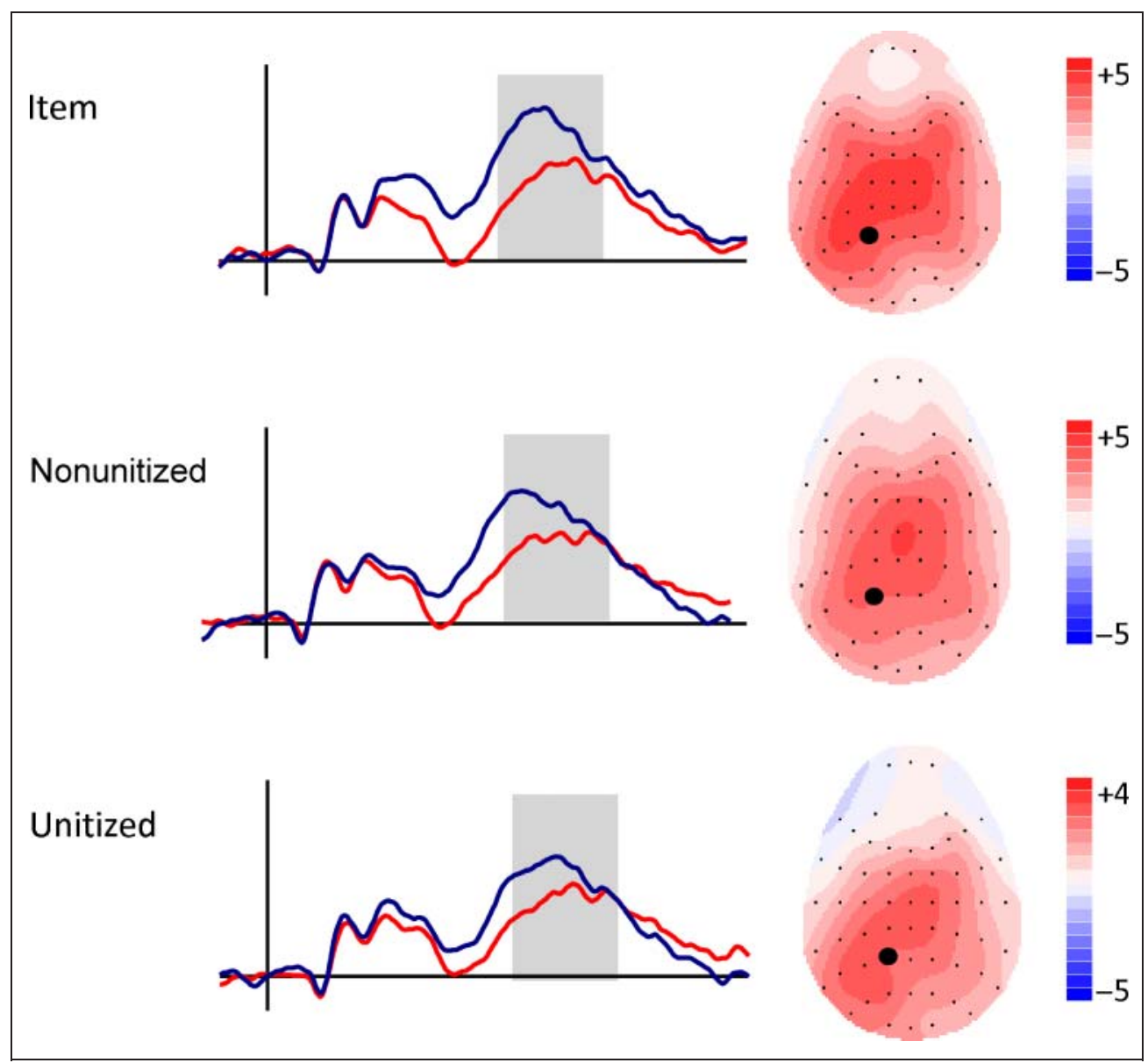


remembered items were originally unitized. The electrophysiological data are clear; as measured by the bilateral frontal old-new effect, the contribution of familiarity to recognition was significantly reduced following unitization. The effect of unitization was also selective; the left parietal old-new effect did not differ in the same way, suggesting that recollection was unaffected by unitization at study. In short, these data provide the first evidence that unitization at encoding incurs "costs" at retrieval, consistent with the "benefits and costs" view. Below, we summarize the theoretical significance of the findings, then considering potential concerns and alternative interpretations of the data, before discussing some wider implications.

Our ERP data provide the first empirical support for the claim that unitization produces a "cost," namely, a reduced contribution of familiarity to retrieval following unitization at encoding. The data are clearly consistent with a theoretical account of unitization that argues for "costs and benefits" (e.g., Rhodes \& Donaldson, 2008; Mayes et al., 2007), rejecting the "benefits-only" view (e.g., Yonelinas et al., 1999). The demonstration of a "cost" at retrieval adds weight to the importance of unitization in characterizing episodic memory (e.g., see Henke, 2010), complementing the previously demonstrated "benefit" of increased familiarity during associative recognition when word pairs are unitized (e.g., Rhodes \& Donaldson, 2007, 2008; Quamme et al., 2007). More broadly, Mayes and colleagues (Mayes et al., 2007) have argued that unitization is only useful as a theoretical concept provided that associations that are agreed to be unitized can be shown to carry measurable cost. From this perspective, examining the ERP correlates of familiarity provides an objective means of assessing whether unitization has actually occurred.

Before considering the ERP data in more detail, however, we first consider an alternative interpretation of the current findings. Put simply, if "costs" occur, why was there no difference in overall recognition performance as a function of unitization? At retrieval, responses in the item condition were faster, more accurate, and better discriminated compared with either the nonunitized or unitized conditions (as per transfer appropriate processing: Morris, Bransford, \& Franks, 1977). By contrast, however, no significant behavioral differences were found between the nonunitized and unitized conditions (see Table 2). On this basis, one might consider the use of the term "cost" to be questionable because there was no direct consequence for memory performance per se. Indeed, the result could even be recharacterized as a "'benefit" because equivalent levels of memory retrieval were achieved through "less" overall neural processing. Although we have some sympathy with this view, here we use the term "cost" because of the theoretical framework that motivated the study. The critical issue is simply that the ERP data reveal a reduction in the contribution of familiarity to recognition.

More broadly, regardless of how it is described, our view is that the ERP data reveal a differential contribution of familiarity to memory because a different kind of information is being retrieved following unitization (i.e., item level information rather than associative information). Rather than mapping retrieval processes on to tasks, unitization data suggest that retrieval processes map onto (are engaged by) particular kinds of representations. In essence, manipulating the way in which word pairs were encoded resulted in a different kind of representation in memory and necessitated the differential engagement of recollection and familiarity when memory was later tested. Importantly, however, we do not consider the unitized condition to be an item recognition test, not at least because the stimuli were random (unrelated) word pairs, not compound words such as blackbird or butterfly. In this context, the presence of equivalent behavioral performance means that we can be confident that differences in the electrophysiological data across unitized and nonunitized conditions are not simply the result of changes in task difficulty or response bias.

Our approach to investigating unitization clearly relies on the use of ERP old-new effects as measures of recollection and familiarity. Here, we consider the two ERP effects in turn, starting with the left parietal old-new effect, which is widely accepted as providing an index of recollection (e.g., see Rugg \& Curran, 2007; Wilding, 2000). As expected, the left parietal effect was present for all conditions, being significantly larger for the item condition $(3.36 \mu \mathrm{V})$ than either nonunitized $(2.75 \mu \mathrm{V})$ or unitized $(2.19 \mu \mathrm{V})$ conditions. The fact that the recollectionrelated ERP effect was smaller for word pairs than for items studied alone most likely reflects the need to encode two words rather than one (within the same overall study time). Although the processing of items in the noninteractive imagery and single item conditions should have been equivalent in kind, the difference in processing load would nevertheless be expected to reduce later recollection for the word pair conditions. The difference in the size of the left parietal effect is also consistent with the fact that overall performance was significantly better for the single-item condition (as discussed above).

Perhaps more puzzling is the finding that the left parietal effect did not differ significantly between the two word pair conditions, suggesting that unitization did not impact on recollection. From a dual-process perspective, the dissociation between the ERP correlates is reasonable given that the processes operate independently in neural terms. How the two processes combine to contribute to performance functionally is unclear, however. Given that overall recognition rates were equivalent for the unitized and nonunitized conditions, an assumption of functional independence (and linear additivity) would predict that a smaller contribution of familiarity would lead to a greater (compensatory) contribution of recollection. If the processes are functionally redundant, however, equivalent recognition rates can be achieved through the contribution of recollection to performance, even when the underlying contribution of familiarity changes. The current 
experiment was not, of course, designed to discriminate between these possibilities, and strong conclusions about the relationship between the two processes are therefore not warranted on the basis of the current findings. Nonetheless, two points are worthy of note. First, we do not view the left parietal findings as a simple null result-if differences in the contribution of recollection existed, we would have expected to see them given that a difference was visible for the item condition. Second, regardless of the functional relationship between recollection and familiarity, existing accounts of unitization predict a selective effect on familiarity, and our ERP data therefore provide support for this view.

Of primary interest here was the early bilateral frontal old-new effect used to measure familiarity. Significant bilateral frontal effects were present for both the item $(2.62 \mu \mathrm{V})$ and nonunitized $(1.71 \mu \mathrm{V})$ conditions. Critically, in contrast, the effect was greatly reduced for the unitized condition (although not entirely absent: $0.65 \mu \mathrm{V}$ ). Here, we interpret the bilateral frontal effect as a correlate of familiarity (e.g., Curran, Tepe, \& Piatt, 2006; Curran, 2000; for a review, see Rugg \& Curran, 2007), but it is important to recognize that alternative views exist. In particular, some authors have linked the bilateral frontal effect to semantic (conceptual) priming rather than familiarity (e.g., see Voss \& Federmeier, 2010; Paller, Voss, \& Boehm, 2007), an alternative view that has been firmly rejected by others (e.g., see Yu \& Rugg, 2010). In the current context, it is unclear how plausible a semantic priming account is, given that all three conditions employed equivalent stimuli, a mental imagery task, and lead to conscious remembering. Thus, although acknowledging that the ERP old-new effects are liable to future reinterpretation, we believe that the current weight of evidence supports the use of the bilateral frontal effect as an index of familiarity.

How then should we interpret the presence of a small bilateral frontal effect following unitization? In theoretical terms, the remaining familiarity is not problematic because the "costs" view only predicts a relative decrease in familiarity, rather than its complete absence. Thus, one interpretation of the data is that familiarity does still contribute to the recognition of component parts following unitization. We highlight an alternative possibility, however. Given that the ERP effects are measured across multiple trials, it seems reasonable to expect some variability at encoding, giving rise to a residual contribution of familiarity. At the very least, we cannot rule out the possibility that some pairs (e.g., words that were more abstract or less familiar to the participants) were not successfully unitized. A pressing task for future studies is, therefore, to examine the effectiveness of unitization task instructions and the potential "leaking in" of familiarity effects elicited by items for which unitization was unsuccessful.

Questions concerning the effectiveness of unitization task instructions also highlight the importance of comparing tasks across studies. For example, what is the relationship between our item versus interactive imagery conditions and the sentence versus compound encoding conditions used elsewhere (e.g., Bader et al., 2010; Haskins et al., 2008; Quamme et al., 2007)? We believe that the critical feature of unitized conditions is that the target words are combined into a single coherent whole. Whether this is accomplished through the use of interactive imagery (as here) or by creating a compound word (as per Quamme et al., 2007) should, we believe, be largely immaterial. Both tasks have the effect of creating a unitized representation (although there may be arguments as to which is more effective in achieving this goal). In contrast, our item imagery condition and the sentence frames used elsewhere both emphasize that target words are encoded as separate entities. The example sentence frames provided in previous studies are clearly designed to ensure that separate representations are formed of each target word (e.g., "The SAUSAGE tasted better than the CIGARETTE," from Bader et al., 2010). Furthermore, in these studies, the instructions also serve to have the target words encoded separately (e.g., separately rate each word as to how well it fits into the sentence frame). By contrast, we would predict that sentence frames promoting interactive imagery (e.g., "The COW tipped over the TABLE") should encourage familiarity for associated word pairs; whether different tasks elicit relative degrees of unitization remains to be seen.

The present study extends our understanding of how and when familiarity and recollection contribute to retrieval. Specifically, the findings suggest that, when multipart stimuli are encoded as a single unit, the individual elements become inaccessible to familiarity, and retrieval must therefore rely primarily on recollection. On this basis, it seems clear that further studies examining both the potential benefits and costs of unitization are warranted. For example, the mechanisms underlying unitization are currently unspecified-how exactly do encoding instructions change the nature of the representation? Are the representations of individual items actively inhibited or simply less strongly activated than the combined representation? Equally, is unitization "all-or-none," or are there "levels of unitization"? More concretely, is the specific encoding task, such as the use of a mediating sentence context, critical? Current findings are insufficient to answer even this question, and given the variety of encoding conditions that might lead to stimuli being processed holistically, comparisons across different encoding tasks are clearly necessary.

Finally, we highlight the broader significance of the current findings. In healthy adults, both recollection and familiarity can contribute to memory retrieval. When recollection is impaired, however, memory necessarily relies on familiarity. For example, patients with damage confined to the hippocampus exhibit selective impairments in recollection (e.g., Vann et al., 2009; Aggleton et al., 2005; Mayes et al., 2004; Yonelinas, 2002). Similarly, patients with Alzheimer disease and its precursor Mild Cognitive Impairment (MCI) both show relatively preserved familiarity in the face of impaired recollection 
(Serra et al., 2010; Gallo, Sullivan, Daffner, Schacter, \& Budson, 2004). Moreover, even in healthy adults, recollection deteriorates with age, whereas familiarity remains relatively preserved (Daselaar, Fleck, Dobbins, Madden, \& Cabeza, 2006; Howard, Bessette-Symons, Zhang, \& Hoyer, 2006; Prull, Dawes, Martin, Rosenberg, \& Light, 2006; Yonelinas, 2002; Jennings \& Jacoby, 1997). Under all of these conditions, it is clearly essential to understand how best to enable familiarity to support episodic retrieval, not least because it may enable us to better tailor rehabilitation or memory training in patients and in healthy aging adults.

\section{Acknowledgments}

D. I. D. and L. K. P. are members of SINAPSE (www.sinapse.ac. uk), a pooling initiative funded by the Scottish Funding Council and the Chief Scientist Office of the Scottish Executive.

Reprint requests should be sent to Lea Pilgrim, Department of Psychology, University of Stirling, Stirling, Scotland, FK9 4LA, United Kingdom, or via e-mail: lea.pilgrim@gmail.com.

\section{REFERENCES}

Aggleton, J. P., Vann, S. D., Denby, C., Dix, S., Mayes, A. R., Roberts, N., et al. (2005). Sparing of the familiarity component of recognition memory in a patient with hippocampal pathology. Neuropsychologia, 43, 1810-1823.

Bader, R., Mecklinger, A., Hoppstädter, M., \& Meyer, P. (2010). Recognition memory for one-trial-unitized word pairs: Evidence from event-related potentials. Neuroimage, 50, $772-781$

Coltheart, M. (1981). The MRC psycholinguistic database. Quarterly Journal of Experimental Psychology, Section A, 33, 497-505.

Curran, T. (2000). Brain potentials of recollection and familiarity. Memory \& Cognition, 28, 923-938.

Curran, T. (2004). Effects of attention and confidence on the hypothesized ERP correlates of recollection and familiarity. Neuropsychologia, 42, 1088-1106.

Curran, T., Tepe, K. L., \& Piatt, C. (2006). Event-related potential explorations of dual processes in recognition memory. In H. D. Zimmer, A. Mecklinger, \& U. Lindenberger (Eds.), Binding in buman memory: A neurocognitive approach (pp. 467-492). Oxford, UK: Oxford University Press.

Daselaar, S. M., Fleck, M. S., Dobbins, I. G., Madden, D. J., \& Cabeza, R. (2006). Effects of healthy aging on hippocampal and rhinal memory functions: An event-related fMRI study. Cerebral Cortex, 16, 1771-1782.

Diana, R. A., den Boom, W. V., Yonelinas, A. P., \& Ranganath, C. (2011). ERP correlates of source memory: Unitized source information increases familiarity-based retrieval. Brain Research, 1367, 278-286.

Donaldson, D. I., \& Curran, T. (2007). Potential (ERP) studies of recognition memory for faces. Neuroimage, 36, 488-489.

Gallo, D. A., Sullivan, A. L., Daffner, K. R., Schacter, D. L., \& Budson, A. E. (2004). Associative recognition in Alzheimer's disease: Evidence for impaired recall-to-reject. Neuropsychology, 18, 556-563.

Graf, P., \& Schacter, D. L. (1989). Unitization and grouping mediate dissociations in memory for new associations.
Journal of Experimental Psychology: Learning, Memory, and Cognition, 15, 930-940.

Haskins, A. L., Yonelinas, A. P., Quamme, J. R., \& Ranganath, C. (2008). Perirhinal cortex supports encoding and familiarity-based recognition of novel associations. Neuron, 59, 554-560.

Henke, K. (2010). A model for memory systems based on processing modes rather than consciousness. Nature Reviews Neuroscience, 11, 523-532.

Hockley, W. E., \& Cristi, C. (1996). Tests of encoding tradeoffs between item and associative information. Memory $\mathcal{E}$ Cognition, 24, 202-216.

Howard, M. W., Bessette-Symons, B., Zhang, Y., \& Hoyer, W. J. (2006). Aging selectively impairs recollection in recognition memory for pictures: Evidence from modeling and receiver operating characteristic curves. Psychology and Aging, 21, 96-106.

Jasper, H. (1958). The ten twenty system of the International Federation. Electroencephalography \& Clinical Neurophysiology, 10, 37-75.

Jennings, J. M., \& Jacoby, L. L. (1997). An opposition procedure for detecting age-related deficits in recollection: Telling effects of repetition. Psychology and Aging, 12, 352-361.

Kiss, G. R., Armstrong, C., Milroy, R., \& Piper, J. (1973). An associative thesaurus of English and its computer analysis. In A. J. Aitken, R. W. Bailey, \& N. Hamilton-Smith (Eds.), The computer and literary studies (pp. 153-167). Edinburgh: University Press.

Mayes, A., Montaldi, D., \& Migo, E. (2007). Associative memory and the medial temporal lobes. Trends in Cognitive Sciences, 11, 126-135.

Mayes, A. R., Holdstock, J. S., Isaac, C. L., Montaldi, D., Grigor, J., Gummer, A., et al. (2004). Associative recognition in a patient with selective hippocampal lesions and relatively normal item recognition. Hippocampus, 14 , 763-784.

McGee, R. (1980). Imagery and recognition memory: The effects of relational organization. Memory $\&$ Cognition, 8, 394-399.

Morris, C. D., Bransford, J. D., \& Franks, J. J. (1977). Levels of processing versus transfer appropriate processing. Journal of Verbal Learning and Verbal Behavior, 16, 519-533.

Paller, K. A., Voss, J. L., \& Boehm, S. G. (2007). Validating neural correlates of familiarity. Trends in Cognitive Sciences, 11, 243-250.

Prull, M. W., Dawes, L. L. C., Martin, A. M., Rosenberg, H. F., \& Light, L. L. (2006). Recollection and familiarity in recognition memory: Adult age differences and neuropsychological test correlates. Psychology and Aging, 21, 107-118.

Quamme, J. R., Yonelinas, A. P., \& Norman, K. A. (2007). Effect of unitization on associative recognition in amnesia. Hippocampus, 17, 192-200.

Rhodes, S. M., \& Donaldson, D. I. (2007). Electrophysiological evidence for the influence of unitization on the processes engaged during episodic retrieval: Enhancing familiarity based remembering. Neuropsychologia, 45, 412-424.

Rhodes, S. M., \& Donaldson, D. I. (2008). Electrophysiological evidence for the effect of interactive imagery on episodic memory: Encouraging familiarity for non-unitized stimuli during associative recognition. Neuroimage, 39, 873-884.

Rugg, M. D., \& Curran, T. (2007). Event-related potentials and recognition memory. Trends in Cognitive Sciences, 11, $251-257$.

Searcy, J. H., \& Bartlett, J. C. (1996). Inversion and processing of component and spatial-relational information in faces. Journal of Experimental Psychology: Human Perception and Performance, 22, 904-915. 
Serra, L., Bozzali, M., Cercignani, M., Perri, R., Fadda, L., Caltagirone, C., et al. (2010). Recollection and familiarity in amnesic mild cognitive impairment. Neuropsychology, 24, 316-326.

Snodgrass, J. G., \& Corwin, J. (1988). Pragmatics of measuring recognition memory: Applications to dementia and amnesia. Journal of Experimental Psychology: General, 117, 34-50.

Vann, S. D., Tsivilis, D., Denby, C. E., Quamme, J. R., Yonelinas, A. P., Aggleton, J. P., et al. (2009). Impaired recollection but spared familiarity in patients with extended hippocampal system damage revealed by 3 convergent methods. Proceedings of the National Academy of Sciences, U.S.A., 106, 5442-5447.

Voss, J. L., \& Federmeier, K. D. (2010). FN400 potentials are functionally identical to N400 potentials and reflect semantic processing during recognition testing. Psychophysiology, $48,532-546$.

Wilding, E. L. (2000). In what way does the parietal ERP old/new effect index recollection? International Journal of Psychophysiology: Official Journal of the International Organization of Psychophysiology, 35, 81-87.

Yonelinas, A. P. (2002). The nature of recollection and familiarity: A review of 30 years of research. Journal of Memory and Language, 46, 441-517.

Yonelinas, A. P., Kroll, N. E., Dobbins, I. G., \& Soltani, M. (1999). Recognition memory for faces: When familiarity supports associative recognition judgments. Psychonomic Bulletin and Review, 6, 654-661.

Yu, S. S., \& Rugg, M. D. (2010). Dissociation of the electrophysiological correlates of familiarity strength and item repetition. Brain Research, 1320, 74-84. 